EPJ Web of Conferences 81, 04006 (2014)

DOI: $10.1051 /$ epjconf/20148104006

(C) Owned by the authors, published by EDP Sciences, 2014

\title{
Exotic meson studies at LHCb
}

\author{
Michal Kreps ${ }^{1, a}$ \\ for the LHCb Collaboration \\ ${ }^{1}$ Department of Physics, University of Warwick, Gibbet Hill Road, Coventry CV4 7AL, UK
}

\begin{abstract}
The latest years have seen a resurrection of interest in searches for exotic states motivated by tantalising observations of several states. Using the $p p$ collisions data collected at 7 and $8 \mathrm{TeV}$ by the LHCb experiment, we performed studies of the $X(3872)$ decay rate to $\psi(2 S) \gamma$ final state, as well as confirmation the $Z(4430)^{+}$state.
\end{abstract}

\section{Introduction}

In 1964, Gell-Mann in his "Schematic model of baryons and mesons" suggested that baryons are constructed from quarks by using combinations $(q q q),(q q q q \bar{q})$ etc., while mesons are made out of $(q \bar{q}),(q q \overline{q q})$, etc. [1]. After 50 years and despite huge experimental effort, until recently we did not had convincing candidate for hadron beyond $(q \bar{q})$ or $(q q q)$ combinations. While some candidates existed, it turned out that convincing evidence for other combinations is extremely difficult to obtain. Latest revival of the quest for hadron states beyond $(q \bar{q})$ or $(q q q)$ combinations started in 2003 with discovery of $X(3872)$ at Belle [2]. Since then many states in charmonium region were observed and for many of them, different interpretations are discussed. In these proceedings, we discuss into details of two measurements, one studying radiative $X(3872)$ decays and the other studying $Z(4430)^{+}$state.

\section{Radiative decays of $\mathrm{X}(3872)$}

The $X(3872)$ state was observed in invariant mass distribution of $J / \psi \pi^{+} \pi^{-}$in $B^{+} \rightarrow J / \psi \pi^{+} \pi^{-} K^{+}$ decays. Its mass did not fit well predicted charmonium spectrum and proximity of its mass to $D^{* 0} \bar{D}^{0}$ threshold and soon after, interpretation as molecule appeared followed by tetraquark interpretation [3]. After 10 years of work to understand this state we learned significant amount of details, including quantum numbers which are determined to be $J^{P C}=1^{++}$[4]. Despite this progress and more than 140 papers published to date, the nature of $X(3872)$ state is still not clear.

One of the quantities, which is sensitive to the nature of $X(3872)$ state is ratio of branching fractions for radiative decays to $\psi(2 S) \gamma$ and $J / \psi \gamma\left(R_{\psi}\right)$. This ratio is predicted to be in the range $(3-4) \times 10^{-3}$ for a $D^{* 0} \bar{D}^{0}$ molecule, $1.2-15$ for a pure $c \bar{c}$ state and $0.5-5$ for a mixture moleculecharmonium mixture (see references in [6]). The decay $X(3872) \rightarrow \psi(2 S) \gamma$ was seen by Babar experiment while Belle experiment did not observed this decay. The central value $R_{\psi}$ reported by Babar collaboration is larger than upper limit set by the Belle experiment [5].

\footnotetext{
ae-mail: M.Kreps@warwick.ac.uk
} 

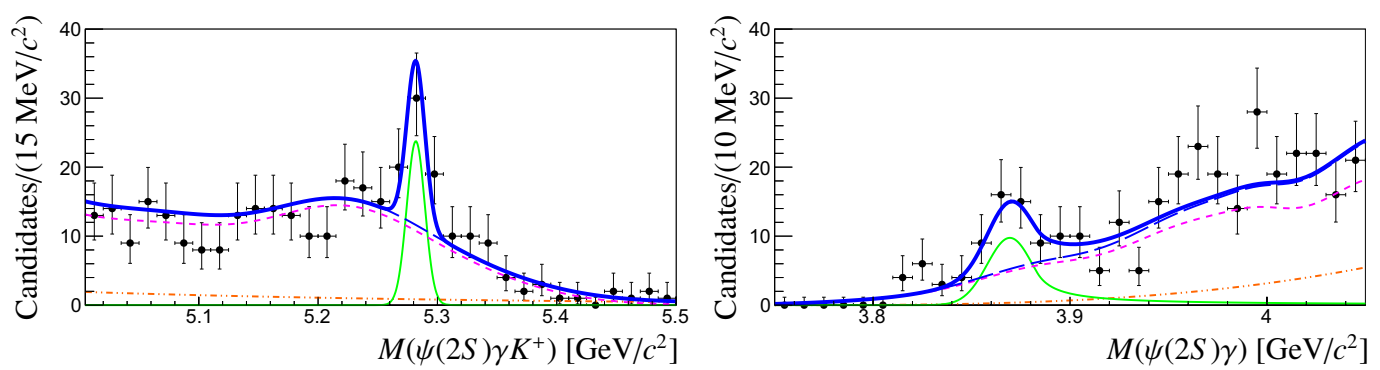

Figure 1. Invariant mass distribution of $\psi(2 S) \gamma K^{+}$combinations (left) and $\psi(2 S) \gamma$ subcombinations. In each case, only events within $\pm 3 \sigma$ around the signal peak in other invariant mass are plotted.

In the $\mathrm{LHCb}$ experiment, we search for the decay $X(3872) \rightarrow \psi(2 S) \gamma$ using $3 \mathrm{fb}^{-1}$ of data with $X(3872)$ produced in the $B^{+} \rightarrow X(3872) K^{+}$decays [6]. The invariant mass distributions of $\psi(2 S) \gamma$ and $\psi(2 S) \gamma K^{+}$combinations are shown in Fig. 1. Clear signal is visible in both of them. The observed signal of $36.4 \pm 9.0$ decays has significance of $4.4 \sigma$. Using this signal, we measure

$$
R_{\psi}=\frac{\mathcal{B}(X(3872) \rightarrow \psi(2 S) \gamma)}{\mathcal{B}(X(3872) \rightarrow J \psi \gamma)}=2.46 \pm 0.64(\text { stat }) \pm 0.29(\text { syst })
$$

While measured $R_{\psi}$ is compatible with $X(3872)$ interpretations as pure $c \bar{c}$ and mixture of $c \bar{c}$ with $D^{* 0} \bar{D}^{0}$ molecule, it is clearly incompatible with pure $D^{* 0} \bar{D}^{0}$ molecule.

\section{Confirmation of $\mathrm{Z}(\mathbf{4 4 3 0})^{+}$}

The game changing discovery was made in 2007 by Belle experiment, which observed peak in the $\psi(2 S) \pi^{+}$invariant mass distribution in $B^{0} \rightarrow \psi(2 S) K^{-} \pi^{+}$decays [7]. As natural width indicated strong decay to $c \bar{c}$ final state, it had to contain the $c \bar{c}$ component, but because of charge, it is clear that it cannot be conventional $c \bar{c}$ state. Subsequent reanalysis of the data with 2D and later 4D amplitude analysis resulted in some change of the measured properties, but basic picture remained the same $[8,9]$. In response to the observation, Babar experiment analysed decays $B^{+, 0} \rightarrow \psi(2 S) \pi^{+} K^{0,+}$ using model independent approach in which question, whether $\psi(2 S) \pi^{+}$invariant mass distribution can be explained by reflections of activity on $K^{-} \pi^{+}$axis of Dalitz plot, was evaluated. In the analysis it was concluded, that there is no need for the state decaying to $\psi(2 S) \pi^{+}$and that the data can be fully described by the $K^{-} \pi^{+}$resonances [10].

In analysis performed by LHCb experiment [11], we use both experimental approaches. Study uses full $\mathrm{LHCb}$ dataset with integrated luminosity of $3 \mathrm{fb}^{-1}$. We reconstruct about $25000 \mathrm{~B}^{0} \rightarrow$ $\psi(2 S) K^{-} \pi^{+}$signal decays with about $4 \%$ background in the signal region. The sample size is about 10 times larger than the ones analysed by B-factories, while the background level is about half. Dalitz plot of the selected events in the signal region is shown in Fig. 2. Clear contributions from $K^{*}(892)$ and kaon states around $1.43 \mathrm{GeV}$ are visible as two vertical bands. The $\mathrm{Z}(4430)^{+}$state would correspond to the horizontal band at $m^{2}\left(\psi(2 S) \pi^{+}\right) \approx 20 \mathrm{GeV}^{2}$.

To build expectation of reflections from $K^{-} \pi^{+}$contributions to $\psi(2 S) \pi^{+}$mass, we measure in the data Legendre moments as a function of the $K^{-} \pi^{+}$invariant mass. Then using observed $K^{-} \pi^{+}$ invariant mass distribution along with moments corresponding to maximal spin 2 we build Dalitz plot distribution and project it on the $\psi(2 S) \pi^{+}$invariant mass. The comparison of reflections to the 

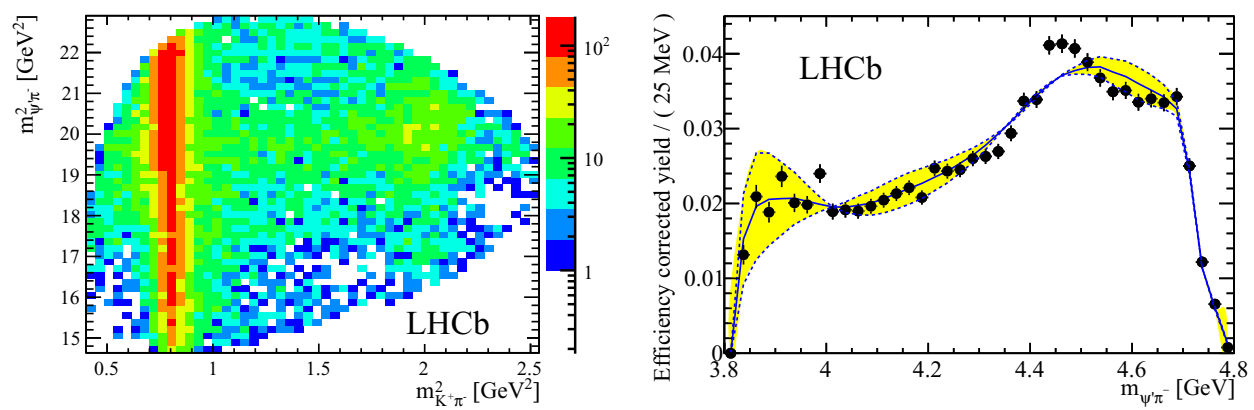

Figure 2. Left: Dalitz plot distribution of the $B^{0} \rightarrow \psi(2 S) K^{-} \pi^{+}$decays. Right: Projection of the $B^{0} \rightarrow$ $\psi(2 S) K^{-} \pi^{+}$Dalitz plot on the $\psi(2 S) \pi^{+}$invariant mass with result of the reflections from $K^{-} \pi^{+}$contributions (blue line with yellow band) as determined using model independent analysis.
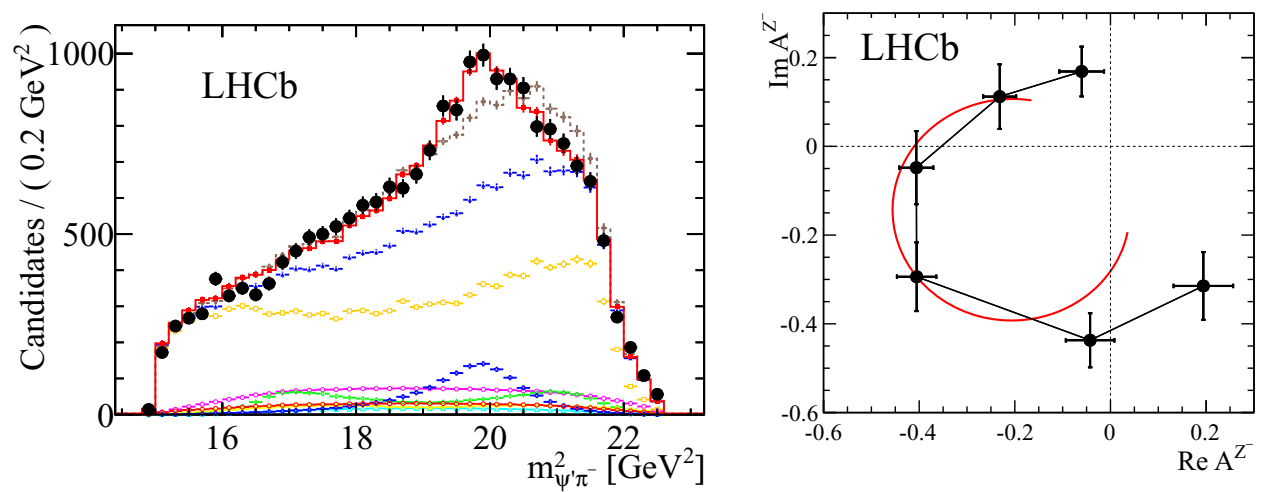

Figure 3. Left: Results of the amplitude fit overlaid over data (point) for the $\psi(2 S) \pi^{+}$invariant mass. Histograms with different symbols represent different contributions to the amplitude. The red histogram shows result of the fit including $Z(4430)^{+}$contribution to the amplitude, while the brown dashed histogram shows result of the fit with only $K^{-} \pi^{+}$contributions. Right: Result of the amplitude fit with $Z(4430)^{+}$contribution included, where the $Z(4430)^{+}$is parametrised in six mass intervals by independent complex amplitudes.

observed data is shown in Fig. 2. It is clear that pure reflections from $K^{-} \pi^{+}$contributions are not sufficient to describe data.

In order to study properties of the $\psi(2 S) \pi^{+}$contribution, amplitude fit is employed. In the fit, two invariant masses squared from the Dalitz plot in Fig. 2 along with muon helicity angle and angle between $\mu^{+} \mu^{-}$and $K^{-} \pi^{+}$planes are fitted. Formalism follows amplitude fit performed by Belle [9]. In the fit, resonances are described by relativistic Breit-Wigner distribution, while description of the angular part uses helicity formalism. The amplitude fit containing only $K^{-} \pi^{+}$contributions yields same conclusion as the model-independent analysis, namely that the data cannot be satisfactorily described. The inclusion of the $\psi(2 S) \pi^{+}$state improves fit quality significantly as can be seen in Fig. 3 . The $\psi(2 S) \pi^{+}$state is consistent with $Z(4430)^{+}$state seen by Belle experiment. As the amplitude fit uses full angular information, it is sensitive to spin, $J$, and parity, $P$ of the $Z(4430)^{+}$state. We test spins 
0,1 and 2 with all possible parities and best fit is achieved with $J^{P}=1^{+}$. The second best hypothesis is $0^{-}$, which is rejected with respect to $1^{+}$assignment by $9.7 \sigma$. From the amplitude fit we measure mass of the $Z(4430)^{+}$state to be $4475 \pm 7$ (stat $)_{-25}^{+15}$ (syst) $\mathrm{MeV}$ and natural width of $172 \pm 13$ (stat) ${ }_{-34}^{+37}$ (syst) $\mathrm{MeV}$. Size of the $Z(4430)^{+}$contribution is evaluated as ratio of integral of square of the $Z(4430)^{+}$ amplitude and integral of square of the full amplitude, which is found to be $5.9 \pm 0.9$ (stat $)_{-3.3}^{+1.5}$ (syst)\% Alternative evaluation which takes ratio of integrals of squares of amplitude with all contributions except of $Z(4430)^{+}$and full amplitude and calculating how much is missing to $100 \%$ yields fraction $16.7 \pm 1.6\left(\mathrm{stat}_{-5.2}^{+2.6}\right.$ (syst)\%. Difference of the two fractions suggests significant interference of the $Z(4430)^{+}$state with $K^{-} \pi^{+}$contributions. The significance of the $Z(4430)^{+}$state is determined to be $13.9 \sigma$. The measured properties are more precise than those from Belle.

To further understand the $Z(4430)^{+}$state, we perform amplitude fit in which $Z(4430)^{+}$BreitWigner is replaced by set of six independent complex amplitudes in $\operatorname{six} \psi(2 S) \pi^{+}$invariant mass intervals. Resulting amplitudes plotted on Argand diagram are shown in Fig. 3. For resonance, amplitudes should show roughly circular behaviour, which is observed in the data. This clearly shows that the observed $Z(4430)^{+}$state is indeed a resonance.

It is worth to note, that in the region of $\psi(2 S) \pi^{+}$mass squared around $16-17 \mathrm{GeV}^{2}$ fit does not fully describe data. Adding second contribution in the $\psi(2 S) \pi^{+}$improves the fit and suggest another state with significance of $6 \sigma$. On the other hand, as the model-independent analysis does not have sensitivity and the Argand diagram is not conclusive, it is clear that this second state is not on the same footing as $Z(4430)^{+}$state.

\section{Conclusions}

With large data sample available at LHCb experiment, spectroscopy of exotic states in the charmonium region can be studied in great details. After many years and measurements for the $X(3872)$ state, the pattern of radiative decays finally starts to exclude some models for the nature of $X(3872)$ state. Examination of the $B^{0} \rightarrow \psi(2 S) K^{-} \pi^{+}$decays for the first time confirms charged $Z(4430)^{+}$state seen by Belle. From study of the phase motion we clearly demonstrate resonance character of this state. The presented measurements demonstrate capabilities of the LHCb experiment for the study of state in the charmonium region and suggest bright future of this research area.

\section{References}

[1] M. Gell-Mann, Phys. Lett. 8, 214 (1964).

[2] S. K. Choi et al. [Belle Collaboration], Phys. Rev. Lett. 91, 262001 (2003).

[3] E. S. Swanson, Phys. Lett. B 588, 189 (2004); L. Maiani, F. Piccinini, A. D. Polosa and V. Riquer, Phys. Rev. D 71, 014028 (2005).

[4] A. Abulencia et al. [CDF Collaboration], Phys. Rev. Lett. 98, 132002 (2007); R. Aaij et al. [LHCb Collaboration], Phys. Rev. Lett. 110, 222001 (2013).

[5] B. Aubert et al. [BaBar Collaboration], Phys. Rev. Lett. 102, 132001 (2009); V. Bhardwaj et al. [Belle Collaboration], Phys. Rev. Lett. 107, 091803 (2011).

[6] R. Aaij et al. [LHCb Collaboration], Nucl. Phys. B 886, 665 (2014).

[7] S. K. Choi et al. [Belle Collaboration], Phys. Rev. Lett. 100, 142001 (2008).

[8] R. Mizuk et al. [Belle Collaboration], Phys. Rev. D 80, 031104 (2009).

[9] K. Chilikin et al. [Belle Collaboration], Phys. Rev. D 88, 074026 (2013).

[10] B. Aubert et al. [BaBar Collaboration], Phys. Rev. D 79, 112001 (2009).

[11] R. Aaij et al. [LHCb Collaboration], Phys. Rev. Lett. 112, 222002 (2014). 